\title{
DE.FORM
}

\author{
Tyson Foster
}

Griffith Film School, Griffith University, South Brisbane, Australia.

t.foster@griffith. edu.au

\begin{abstract}
Concept Statement. Breathing life into the digital void is the core goal of my practice. As a technical artist I use 3D software and interactive real-time graphics to generate responsive performance for sty lized 3D characters in virtual worlds. Working with stylized form allows me to explore the essence of motion. This practice is a symbiosis of code and aesthetics. Virtual Reality (VR) has [re]emerged as an artistic medium that fuses game mechanics and interactivity with active storytelling and character performance. The tranquil first person experience in DE.FORM presents the player with various creatures floating around the player while tracking the players gaze and head position. DE.FORM examines the importance of player immersion and how presence is maintained while interacting with responsive stylized 3D characters in VR. This has been achieved by employing rules of non-verbal communication and plausible motion in order to maintain player immersion.
\end{abstract}

Keywords: virtual reality; rigging; motion; immersion; presence.

\section{$1 \quad$ Exhibition Details}

This proposed artwork is an exploration into gesture, organic motion, and algorithms used to create a sense of empathy with stylized procedural creatures. Working with VR and procedural generation techniques, I create amorphous digital creatures that I use to explore the link between the materiality of virtual form and anatomical deformation derived from the natural world. My practice is VR based and I wish to share this experimental medium with as many people as possible. VR transports the viewer to other worlds that appear as perceptually real to the player. This phenomenon is known as presence ${ }^{1}$, it is this sense of presence which makes VR a powerfully immersive experience.

By utilizing virtual reality as an artistic medium, this work challenges the audience's prior assumptions of the real and the virtual, the corporeal and the incorporeal, and

1 "A variety of stimuli provided by a virtual reality system can cause the user to perceive that s/he is moving through and interacting with the environment created by the technology rather than the user's actual physical environment. Because a virtual reality system provides immersive, high resolution, three-dimensional images and high fidelity, dimensional sound, a virtual reality system can cause the viewer to perceive that $\mathrm{s} /$ he is in an environment that looks and sounds as the viewer believes it does or would in the physical world" [1]. 
audience as observer and audience as participant ${ }^{2}$. Based on Laurel's explanation of interface as theater she explains that the virtual world is created by populating the virtual world with representations of human and computer-generated agents to create an authentic feeling virtual space [2].

\subsection{Play}

In DE.FORM the audience is invited to navigate around various virtual spaces that showcase various procedurally created and animated stylized creatures.

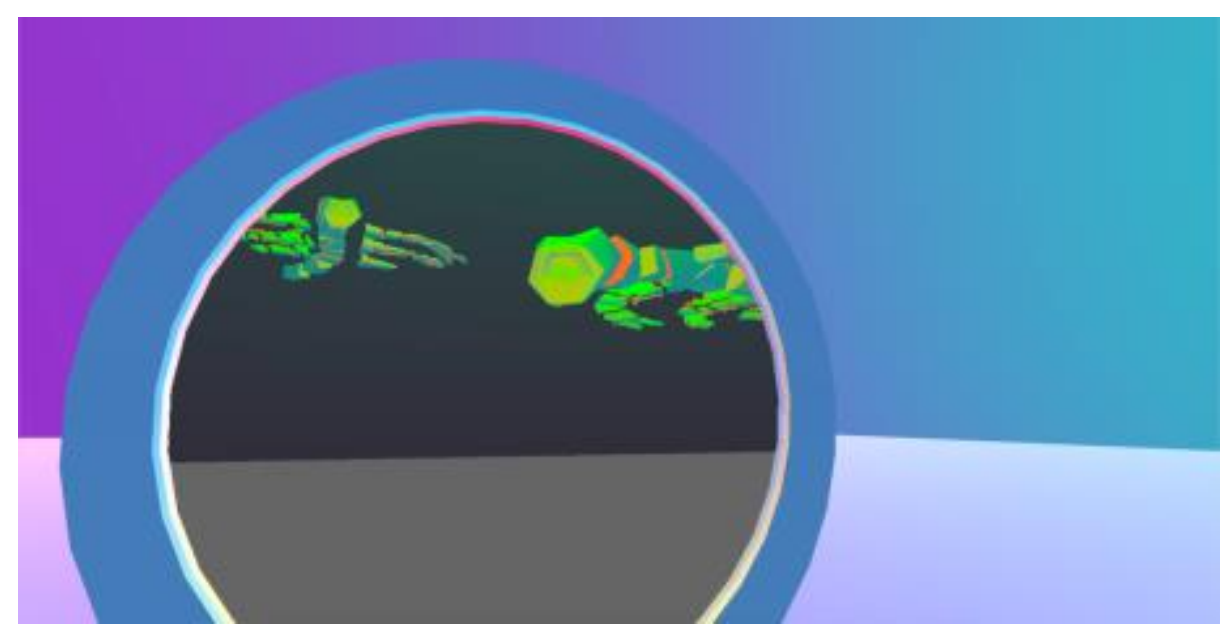

Fig. 1. Player looking through portal gateway to another environment containing two flying creatures on patrol in their own world.

Each space is connected using a portal gateway that allows observation of the next space before committing to enter (Fig. 1). Once the player has teleported to the new game space, the creatures will begin to engage with the player.

\subsection{Mechanics}

The mechanics of the interactions are based around game worlds that are fundamentally changed with the use of VR. The experiences are presented as series a short gameplay atoms ${ }^{3}$. These gameplay atoms create a game-like environment to showcase the procedural creatures in context, without the requirement for complex gameplay sequences.

2 Brenda Laurel explains, “[p] ]eople who are participating in the representation aren't audience members any more. It's not that the audience joins the actors on the stage; it's that they become actors - and the notion of "passive" observers disappears"[2]. 


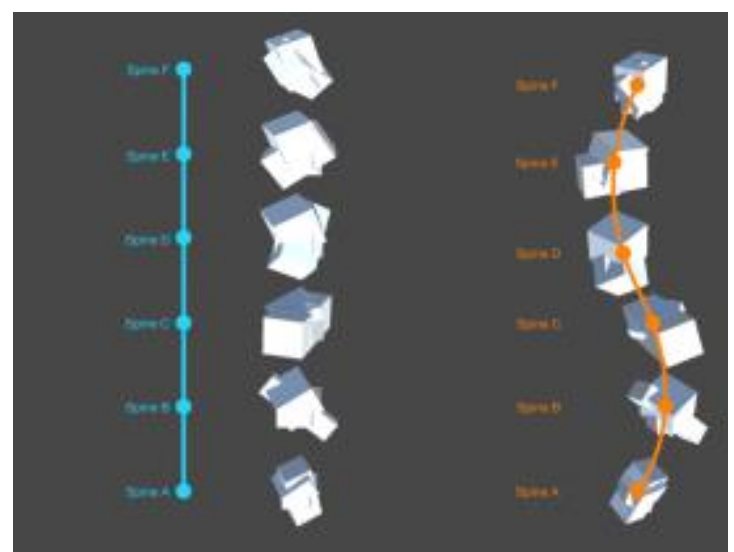

Fig. 2. Top view of abstract spine joint hierarchy created in Unity with unshaded mesh (left) same joint hierarchy with a serpentine animation applied (right).

This above example (Fig. 2) allows for animation to propagate along a character's joint chain such as the spine or limbs, enabling motion to be generated and performed by the procedural character's mesh.

\subsection{Interaction}

Inspired by VR demos such as Showdown Cinematic VR Demo (2015) by Epic, Lost (2015) and Henry (2015) by Oculus Story Studio, and Sightline: The Chair (2013) by Frooxius, interactions in DE.FORM are kept simple, utilizing tap-based input for teleport locomotion with gaze-based input for the interactions with the characters. Laurel suggests that players learn how to interact in the game world by playing with the interactions and observing what happens as a result [2]. Therefore, the interactions in DE.FORM are kept simple in order to facilitate playful exploration of the gaze-based input with the creatures.

By implementing gaze-based and motion-based inputs players utilize head tracked interaction based on their own body movement as the input in the virtual world without abstracting any of the interactions through the use of controller buttons or sticks. This is an attempt to allow audience of varying degrees of prior game experience to naturally interact with the virtual world in order to sustain the perceptual realis $\mathrm{m}^{4}$ of the VR experience.

Each creature reacts to different and surprising ways to the player's gaze and the player avatar's physical presence within the virtual world. The creature reacts to the

4 "Sensory presence," "perceptual realism," "naturalness," "ecological validity", and "tactile engagement" occur when part or all of a person's perception fails to accurately acknowledge the role of technology that makes it appear that s/he is in a physical location and environment in which the sensory characteristics correspond to those of the physical world, i.e., s/he perceives that the objects, events, and/or people s/he encounters look, sound, smell, feel, etc. as they do or would in the physical world [1]. 
player's gaze, such as acknowledging eye contact, and also the player's physical presence in the game world (Fig. 3). Each experience is crated to give a unique perspective on the different creatures that have been created, ranging from passive behaviors to aggressive predatory behavior.

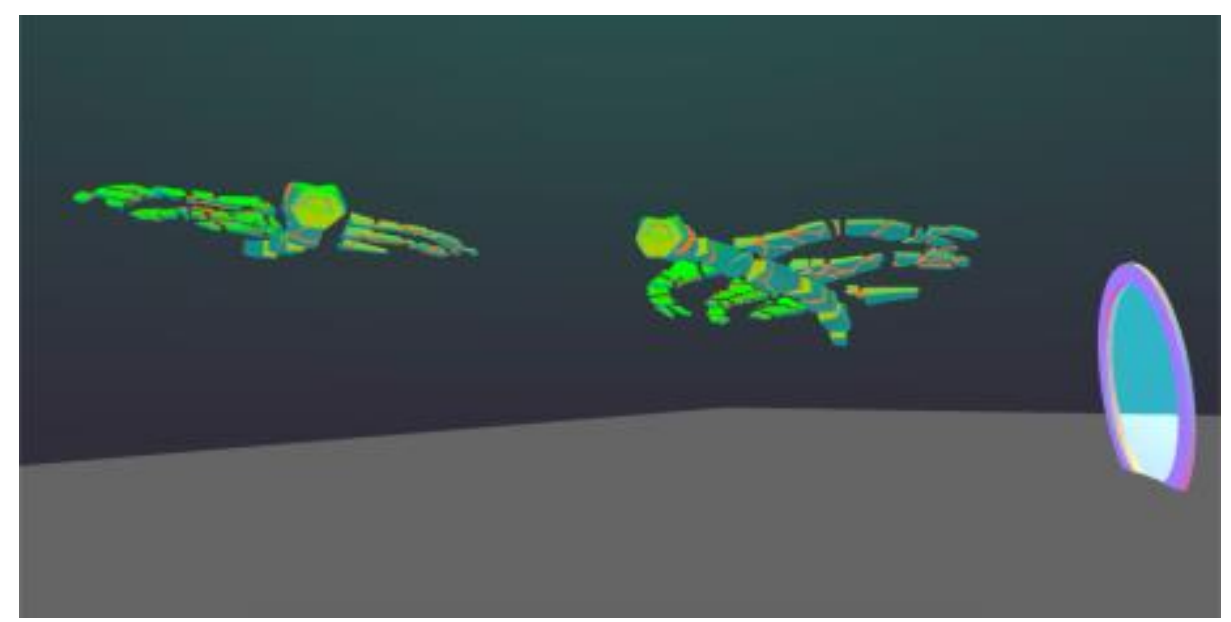

Fig. 3. Creatures reacting to both the player's gaze and the player's position in the world.

\section{References}

1. International Society for Presence Research: The Concept of Presence: Explication Statement. https://ispr.info/ (2000)

2. Laurel, B: Computers as Theatre, pp. 17-18. Addison-Wesley, Massachusetts (1993) 